\title{
Die Bestimmung der klimatischen Schneegrenze in den Schweizer Alpen*
}

\author{
Hermann Escher
}

\section{Zum Begriff der klimatischen Schneegrenze}

Die Zahl der Artikel über die Schneegrenze und deren Bestimmung ist ziemlich groß. Beinahe ebenso groß ist die Zahl der verschiedenen Auffassungen des Schneegrenz-Begriffes. Gute Zusammenstellungen dazu finden sich bei Zeller (1892), v. Klebelsberg (1948/1949) oder bei Messerli (1967).

Der vorliegenden Arbeit wurde die Definition Zinggs (1954) zugrunde gelegt: «v. Klebelsberg (1948/1949) definiert die Schneegrenze in seinem Handbuch wie folgt: ,Die Schneegrenze ist die Linie, jenseits der im Verlauf längerer Zeit mehr Schnee fällt als abschmilzt oder verdunstet.' In dieser Definition kommt dem Zeitbegriff eine gewisse Rolle zu. Ist der Zeitraum auf eine Jahreszeit oder ein Jahr bemessen, so spricht man von temporärer Schneegrenze. Betrachtet man den Zeitraum einiger Jahre oder gar Jahrzehnte, so handelt es sich um die klimatische Schneegrenze unter der Bedingung, $\mathrm{da} ß$ sich die Beobachtungen auf ein horizontales Feld normaler Exposition beziehen. Die klimatische Schneegrenze liegt in jener Höhenlage, in welcher im Mittel einiger Jahre oder Jahrzehnte die Schneedecke 365 Tage dauert...» Das Feld (ca. $30 \mathrm{x}$ $30 \mathrm{~m}$ ) darf also nicht vergletschert sein.

\section{Faktoren, welche die Lage der klimatischen Schneegrenze beeinflussen}

Die Lage der klimatischen Schneegrenze hängt von einer ganzen Reihe verschiedener Faktoren ab (vgl. Streiff-Becker, 1947; v. Klebelsberg, 1948/1949; Seiffert, 1950; Hoinkes, 1952/1953, 1955; Zingg, 1965). Die wichtigsten seien hier ausführlicher dargestellt:

\section{a) Temperatur}

Sowohl für den Auf- wie für den Abbau der Schneedecke ist die Temperatur von entscheidender Bedeutung. Temperaturen über $0^{\circ} \mathrm{C}$ bewirken, daß die Niederschläge in Form von Regen fallen (wobei die Grenze i. a. nicht exakt bei $0^{\circ} \mathrm{C}$ liegt). Anderseits ist das Schmelzen von Schnee nur bei Temperaturen von über $0^{\circ} \mathrm{C}$ möglich. Man müßte daher eigentlich nur die positiven Temperatursummen betrachten $(=$ "Summe aller positiven Tagesmittel der Lufttemperatur innerhalb eines gewissen Zeitraumes», Kuhn, 1958, p. 335). Weil sich aber eindeu- tig nachweisen ließ, daß zwischen den Temperatursummen pro Monat und den Monatsmitteltemperaturen eine sehr hohe Korrelation besteht $(\mathrm{r}=$ +0.98 , Escher, 1969), darf man ohne weiteres Monatsmitteltemperaturen verwenden und braucht die positiven Temperatursummen gar nicht erst $\mathrm{zu}$ berechnen 1 .

Die Temperaturen werden $2 \mathrm{~m}$ über Boden in den normalen Wetterhütten des klimatologischen Netzes der MZA gemessen. Zumindest für das Abschmelzen ist aber die Temperatur an der Schneeoberfläche maßgebend. Es wäre daher zu untersuchen, welcher systematische Fehler damit begangen wird, daß diese Hüttentemperaturen und nicht Schneeoberflächlichen-Temperaturen angewendet werden.

\section{b) Niederschläge}

Sicher beeinflußt die Summe der Winterniederschläge die Dauer der Schneedecke: Je mehr Schnee fällt, um so mehr Zeit braucht es bei einer gegebenen Temperatur, bis er weggeschmolzen ist. Der Regenanteil der Sommerniederschläge wirkt nur gering auf die Zahl der Tage mit Schneedecke ein (vgl. die Messungen von Hoinkes, 1955). Nicht einfach sind dagegen die Auswirkungen von Sommerschneefällen zu erfassen, die in der Regel eine sehr starke Erhöhung der Albedo bewirken. Daher geht man zum Teil sogar so weit, daß man ihnen ein größeres Gewicht zumißt als den Winterschneefällen (Hoinkes, 1968).

Zwischen den Jahresmitteltemperaturen und den Niederschlagsmengen pro Jahr besteht ein gewisser Zusammenhang, der sich numerisch allerdings nicht sehr stark ausdrückt: $r=-0.4$ für 35 Schweizer Stationen und die Jahre 1964-1966 (Escher, 1969). Falls die Niederschläge ziemlich gleichmäßig über das ganze Jahr verteilt sind, laufen höhere Niederschlagsmengen mit erhöhter durchschnittlicher Bewölkung parallel, was in einer Verminderung der Temperatur resultiert.

* Zusammenfassung einer Diplomarbeit, die am Geographischen Institut der Universität Zürich ausgeführt wurde. Die Karte "Klima und Wetter» des Atlas der Schweiz (Lieferung V) wird die hier diskutierten Werte für die Höhe der klimatischen Schneegrenze enthalten. 


\section{c) Wind}

Ambühl (1961) weist darauf hin, daß «durch die Wirkung der Winde Gipfel und Paßlagen hinsichtlich aperer Zeit profitieren». Das gleiche gilt auch für ausgesprochene Luvhänge.

$\mathrm{Da}$ aber den vorliegenden Untersuchungen horizontale Versuchsfelder mit normaler Exposition zugrunde liegen, fällt der Windeinfluß weniger ins Gewicht.

\section{d) Exposition}

Auch da wird versucht, die Einflüsse mit der Bedingung der horizontalen Versuchsfelder zu vermindern.

\section{e) Unterlage der Schneedecke}

Nach Zingg (1954) ist für die Bestimmung der klimatischen Schneegrenze von unvergletschertem Gebiet auszugehen. Auf einer Firn- oder Eisschicht hält sich frisch gefallener Schnee viel länger als auf dem Erdboden. Ungeklärt ist die Frage, wie sich schneefreie, aber gefrorene Böden auswirken. Verhältnisse, die wahrscheinlich ziemlich oft auftreten.

\section{f) Höhenlage}

Abgesehen von Inversionslagen sind Temperaturen und Höhe stark miteinander korreliert: $\mathrm{r}=-0.95$ (Escher, 1969).

Es zeigt sich also deutlich, daß in den Alpen die Lage der klimatischen Schneegrenze vor allem von der Temperatur (Jahresmittel) und den Niederschlägen (Jahressumme in $\mathrm{mm}$ Wasser) abhängt. Von weiteren Einflüssen muß vorerst - was sich ohne weiteres verantworten läßt - abgesehen werden, da sie einmal schwer zu erfassen sind (z. B. Windeinfluß) und zudem zum Teil stark miteinander zusammenhängen oder mit anderen Elementen verbunden sind. Die Temperatur darf geradezu als "Sammelfaktor» angesehen werden, welcher über die Energiebilanz, aber auch (bis zu einem gewissen Grade wenigstens) über Niederschläge und Bewölkung Auskunft zu geben vermag. Wahrscheinlich haben diese Zusammenhänge nur in unserem Klimabereich Gültigkeit, wie z. B. Seiffert (1950) und Messerli (1967) zeigen.

\section{Grundlagen des verwendeten Modells}

Aufgrund dieser Úberlegungen wurden die Ideen von v. Sonklar (1864, zit. in Zeller, 1892), Zingg (1954) und Hess (1967) weiter verfolgt. Sie bauen voll und ganz auf klimatologischen Grundlagen auf und wenden sich gegen die Verwendung von Gletschern und Firnfeldern, da «eine große Zahl von Gletschern nicht unserem Klima angehört, sondern als Relikte zu betrachten sind" (Zingg,
1954). Weitere Fehlerquellen ergeben sich zudem daraus, daß diese Berechnungen auf topographischen Karten erfolgen, die nie bis auf den neuesten Stand der Gletscher nachgeführt sind. Deshalb hat Finsterwalder (1952/1953, zit. in Zingg 1954) Luftbilder verwendet. Es gelang Zingg $(1954,1965)$, eine Beziehung zwischen der Gesamtdauer der zusammenhängenden winterlichen Schneedecke und der Meereshöhe zu finden (einzelne Schneedeckentage im Sommer bleiben unberïcksichtigt). Daraus kann er die Höhenlage der klimatischen Schneegrenze berechnen. Ähnlich geht auch Hess (1967) vor.

Für das vorliegende Modell dienten folgende Unterlagen:

Stationsnetz: $\mathrm{m}$ ü. $\mathrm{M}$.

\begin{tabular}{|c|c|c|}
\hline $\begin{array}{l}\text { Vättis } \\
\text { Davos } \\
\text { Arosa } \\
\text { Weißfluhjoch } \\
\text { (Versuchsfeld }\end{array}$ & $\begin{array}{r}948 \\
1588 \\
1818 \\
2667 \\
2540)\end{array}$ & $\begin{array}{l}\text { Periode 1957-1966. In } \\
\text { der Folge werden diese } \\
\text { Stationen als «Mittelbün- } \\
\text { den» zusammengefaßt }\end{array}$ \\
\hline $\begin{array}{l}\text { Grindelwa!d } \\
\text { Guttannen } \\
\text { Grimsel }\end{array}$ & $\begin{array}{l}1040 \\
1058 \\
1950\end{array}$ & $\begin{array}{l}\text { Periode 1958-1966 } \\
\text { Berner Oberland }\end{array}$ \\
\hline $\begin{array}{l}\text { Visp } \\
\text { Montana } \\
\text { Zermatt }\end{array}$ & $\begin{array}{r}658 \\
1510 \\
1636\end{array}$ & $\begin{array}{l}\text { Periode 1959-1966 } \\
\text { Wallis }\end{array}$ \\
\hline
\end{tabular}

Für jede dieser Stationen stehen aus den Annalen der MZA (1864 ff) und aus unveröffentlichter Originalliteratur (Beobachtungen der Wetter- und Klimastationen) die Jahresmitteltemperatur, die Jahressumme der Niederschläge (in $\mathrm{mm}$ Wasser) und die Jahressumme der Tage mit Schneedecke zur Verfügung. "Eine Schneedecke wird dann angenommen, wenn mindestens die Hälfte der Bodenfläche in der Umgebung der Station um $07.30 \mathrm{mit}$ Schnee bedeckt war» (Annalen der MZA, seit 1949).

Temperaturmittel und Niederschlagsdaten sind über lange Zeiträume hinweg greifbar (seit 1864). Mit der systematischen Erfassung der Schneedekkentage begann die MZA erst 1959; seit 1964 liegen die Beobachtungen für alle Stationen des Netzes vor. Es war daher nicht einfach, Stationen mit ausreichenden Unterlagen zu finden. Die Wahl der 10 obengenannten Stationen wurde weitgehend durch diese Schwierigkeit bestimmt.

\section{Aufbau des Modells}

Temperatur und Niederschlag sind die Hauptelemente, die den Auf- und Abbau der Schneedecke beeinflussen. Daher werden in einem dreidimensionalen Koordinatensystem für die gewählten Statio- 


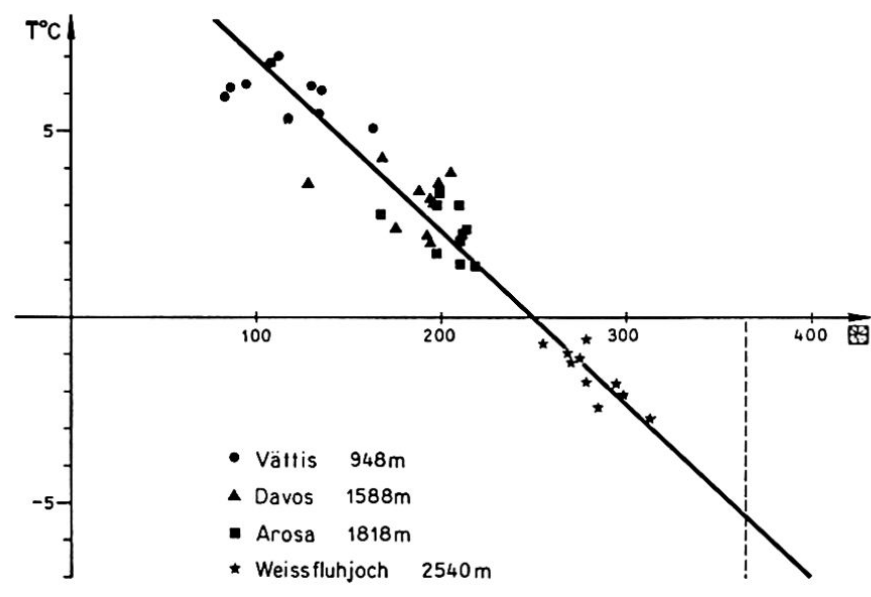

Fig. 1

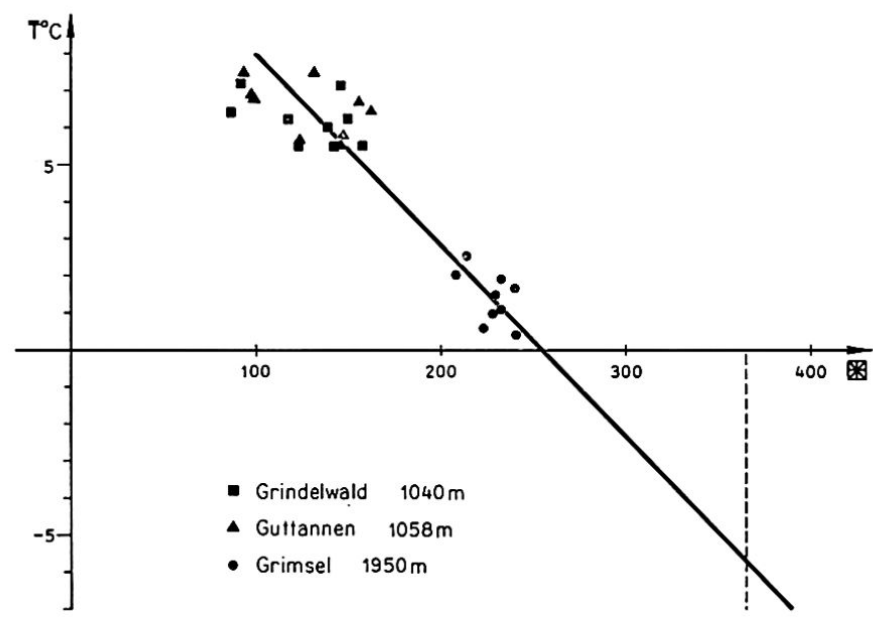

Fig. 3

nen die Jahressumme der Tage mit Schneedecke als abhängige, die Jahresmitteltemperatur und die Jahressumme der Niederschläge (in $\mathrm{mm}$ Wasser) als unabhängige Variablen aufgetragen.

In der Literatur wird darauf hingewiesen, da $\beta$ man mit Sommertemperaturen zu arbeiten habe (Ahlmann, 1924; Seiffert, 1950; Messerli, 1967). Das wirft aber verschiedene Fragen auf: Die Abschmelzperiode ändert mit der Höhe. Müßte man also das Zeitintervall dieser Verschiebung anpassen (z. B. für Stationen auf $2000 \mathrm{~m}$ ü. M. die Temperatur von April bis Mai, auf $3000 \mathrm{~m}$ ü. M. von Juli bis August gebrauchen)? Das gleiche Problem stellt sich auch bei den Niederschlägen: Während in tieferen Lagen der Abbau der Schneedecke schon eingesetzt hat, dauert in der Höhe deren Aufbau noch an. All diese Verschiebungen im Modell auch noch berücksichtigen zu wollen, scheint sehr problema-

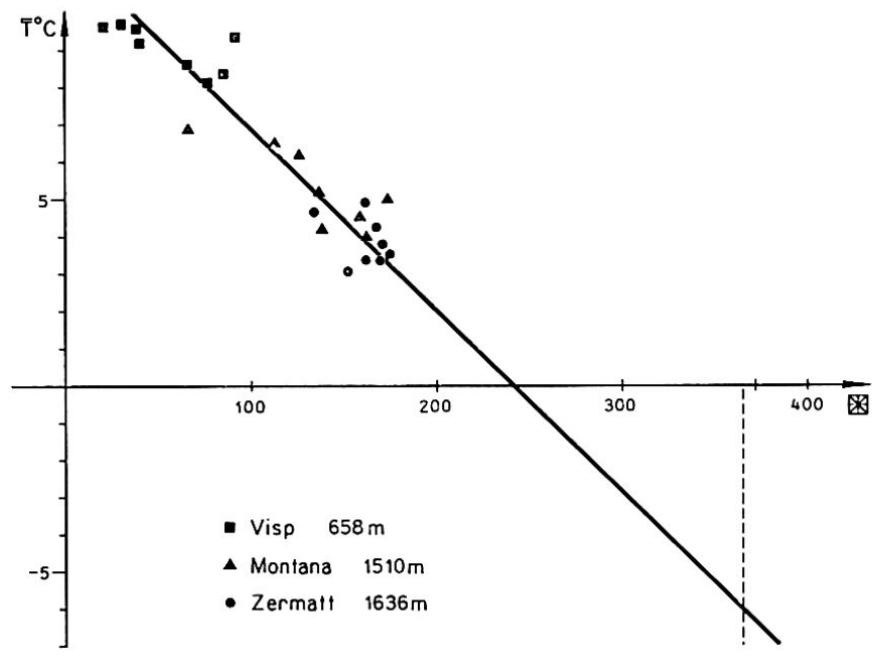

Fig. 2

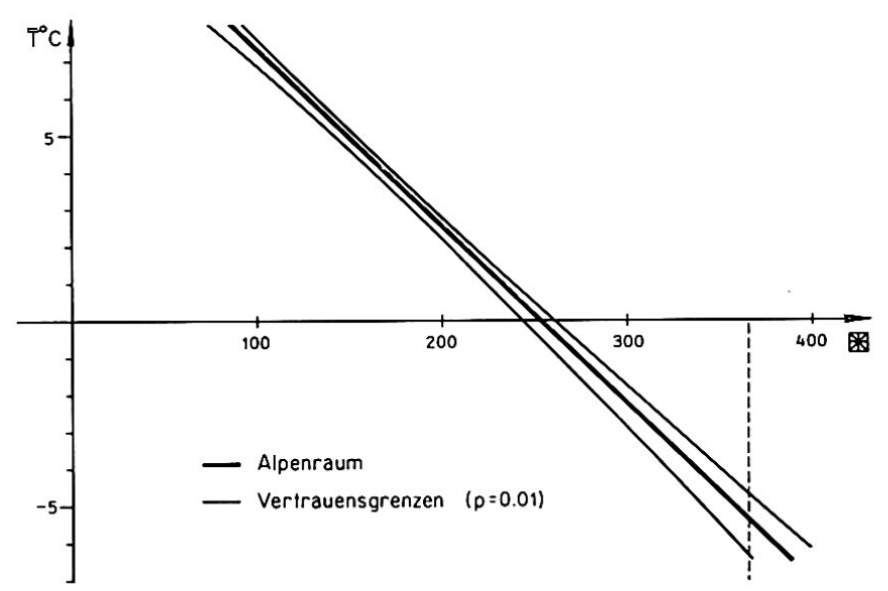

Fig. 4

tisch und schwierig. Es wurden daher nur Jahreswerte verwendet.

\section{Berechnung des Modells}

Die Berechnungen, welche mit dem Computer IBM 360-40 des Rechenzentrums der Universität Zürich ausgeführt wurden, basieren auf der Korrelationsund Regressionsrechnung. Da sie in der Literatur ausführlich dargestellt wird, erübrigt es sich, hier näher darauf einzugehen (vgl. Linder, 1960; Weber, 1967; Kreyszig, 1968).

Vorerst gelangten die drei Regionen getrennt zur Untersuchung. In das genannte Koordinatensystem wird für jede Station und jedes Jahr ein Wert eingetragen, das ergibt - für den Fall Mittelbündens (1957-1966) — im ganzen 4 Punktwolken zu je 
10 Punkten. Die Regressionsrechnung bestimmt nun die Gleichung derjenigen Fläche, die für die Zahl der Tage mit Schneedecke (= abhängige Größe) minimale Abweichungen ergibt. Ist diese Fläche bekannt, läßt sie sich durch Veränderung der Temperatur- und Niederschlagswerte so weit extrapolieren, bis man für die abhängige Variable 365
Schneedeckentage erhält. Man kennt somit die Temperatur- und Niederschlagsbedingungen, die erfüllt sein müssen, damit der Schnee das ganze $\mathrm{Jahr}$ über liegen bleibt. Diesem Temperaturwert kann die ihm entsprechende Höhe zugeordnet werden: Die Höhenlage der klimatischen Schneegrenze ist bestimmt.

\section{Interpretation der vorläufigen Resultate; Einfluß des Niederschlages}

Es wurde also - für jede der drei Regionen einzeln — folgende Gleichung bestimmt:

Zahl der Tage mit Schneedecke $=a+b \cdot R R+c \cdot \bar{T}$

$(\overline{\mathrm{T}}=$ Jahresmitteltemperatur, $\mathrm{RR}=$ Jahressumme der Niederschläge $)$ wobei:

\begin{tabular}{|c|c|c|c|}
\hline \multirow[b]{2}{*}{ Region } & \multirow[b]{2}{*}{ Koeffizienten } & \multicolumn{2}{|c|}{ t-Wert } \\
\hline & & berechnet & $\begin{array}{l}\text { nach Tabelle } \\
(P=0.01)\end{array}$ \\
\hline Mittelbünden & $\begin{array}{l}a=243.07 \\
b=0.01 \\
c=-21.00\end{array}$ & $\begin{array}{r}0.39 \\
-19.50\end{array}$ & $\begin{array}{l}2.70 \\
(n=38)\end{array}$ \\
\hline Berner Oberland & $\begin{array}{l}a=221.62 \\
b=\quad .0 .01 \\
c=-17.65\end{array}$ & $\begin{array}{r}1.35 \\
-8.18\end{array}$ & $\begin{array}{l}2.78 \\
(n=25)\end{array}$ \\
\hline Wallis & $\begin{array}{l}\mathrm{a}=207.02 \\
\mathrm{~b}=0.03 \\
\mathrm{c}=-18.91\end{array}$ & $\begin{array}{r}1.51 \\
-9.20\end{array}$ & $\begin{array}{l}2.82 \\
(\mathrm{n}=22)\end{array}$ \\
\hline
\end{tabular}

Weil es sich bei den beobachteten Werten, die der Regressionsrechnung zugrunde liegen, um eine Auswahl aus einer Grundgesamtheit handelt, ist in jedem Fall zu prüfen, ob die errechneten Koeffizienten signifikant von 0 verschieden sind oder nicht. Man benützt dazu den t-Test (Linder, 1960). Uberschreitet der berechnete t-Wert den tabellierten, so darf bei einer bestimmten Irrtumswahr-

$\begin{array}{ll}\text { Region } & \text { Koeffizient } \\ \text { Mittelbünden } & \mathrm{a}=249.52 \\ & \mathrm{c}=-21.11 \\ \text { Berner Oberland } & \mathrm{a}=254.32 \\ & \mathrm{c}=-19.64 \\ \text { Wallis } & \mathrm{a}=242.01 \\ & \mathrm{c}=-20.51\end{array}$

Zur Stützung dieses Ergebnisses wurden partielle Korrelationskoeffizienten berechnet (für alle drei Gebiete sind sie etwa gleich, Escher, 1969):

Zahl der Schneedeckentage - Temperatur: -0.9 (Niederschlag $=$ konst.) scheinlichkeit $\mathrm{P}$ angenommen werden, daß der Koeffizient signifikant von 0 abweicht.

Wird nun dieser Test auf die oben angegebenen Werte angewendet, so zeigt sich, daß wohl der Koeffizient $\mathrm{c}$ gesichert ist, $\mathrm{b}$ dagegen nur zufällig von 0 abweicht $(P=0.01)$. Somit läßt sich Gleichung (1) reduzieren zu:

Zahl der Tage mit Schneedecke $=a+c \cdot \overline{\mathrm{T}}$ wobei:

t-Wert

berechnet

nach Tabelle

$(\mathrm{P}=0.01)$

$\begin{array}{ll}-20.55 & 2.70\end{array}$

$(\mathrm{n}=38)$

$-12.36$

2.78

$(\mathrm{n}=25)$

$-11.30$

2.82

$(\mathrm{n}=22)$

Zahl der Schneedeckentage - Niederschlag: +0.3 (Temperatur $=$ konst.)

Die Streuungszerlegung ergibt ein entsprechendes Bild. 
Diese Angaben untermauern die These, daß die Niederschläge auf die Zahl der Tage mit Schneedecke keinen wesentlichen Einfluß ausüben.

Man darf daher den Schluß ziehen, daß in den untersuchten Regionen die Zahl der Schneedeckentage in allererster Linie von der Jahresmitteltemperatur abhängt. Sicher vernachlässigt man damit etliche Faktoren, deren Bedeutung aber nicht sehr wesentlich sein kann und die zudem noch schwer zu erfassen sind. Für Detailuntersuchungen, die sich nur über ein kleines Gebiet erstrecken, müßten solche Einflüsse auch betrachtet werden. Hier er- hebt sich dann aber auch die Frage der Versuchsanordnung, wie sie bei der Temperatur diskutiert wurde, viel schärfer.

Es muß deutlich darauf hingewiesen werden, daß dieses Überwiegen des Temperatureinflusses wahrscheinlich nur im Alpenraum Gültigkeit besitzt. Seiffert (1950) und Messerli (1966) zeigen, wie Jahresniederschläge von weniger als $500 \mathrm{~mm}$ die Schneegrenzlage stark beeinflussen.

Gleichung (2) gibt an, bei welcher Jahresmitteltemperatur $\overline{\mathrm{T}}$ der Schnee das ganze Jahr über liegen bleibt²:

$$
\begin{array}{ll}
\text { Mittelbünden: } & \overline{\mathrm{T}} \text { an der Schneegrenze: }-5.5^{\circ} \mathrm{C}(1957-1966) \\
\text { Berner Oberland: } & \overline{\mathrm{T}} \text { an der Schneegrenze: }-5.7^{\circ} \mathrm{C}(1958-1966) \\
\text { Wallis: } & \overline{\mathrm{T}} \text { an der Schneegrenze: }-6.0^{\circ} \mathrm{C}(1959-1966)
\end{array}
$$

\section{Vergleich der drei Regionen}

Schon die graphischen Darstellungen lassen vermuten, daß die Regressionskoeffizienten der drei Gebiete sich nicht signifikant voneinander unterscheiden. Das entsprechende Testverfahren (Linder, 1960) wurde angewendet, und es bestätigte die Vermutung voll und ganz: Die Abweichungen der Re- gressionskoeffizienten voneinander sind nur zufälliger Natur.

Daher wurden die drei Regionen zusammengefaßt und gemeinsam verarbeitet (Periode 1959-1966): Zahl der Tage mit Schneedecke $=a+c \cdot \bar{T}$ wobei:

$$
\begin{array}{lrl}
\mathrm{a}=250.92 & t \text {-Wert }: \text { berechnet } & \ldots \text { nach Tabelle } 2.64 \\
\mathrm{c}=-20.88 & -29,49 & (\mathrm{n}=78, \mathrm{P}=0.01)
\end{array}
$$

Jahrestemperatur an der Schneegrenze: $-5.5^{\circ} \mathrm{C}$. Dieser Wert dürfte im ganzen Alpenraum gültig sein.

Es fragt sich allerdings, ob es richtig ist, die drei Ge- biete so zusammenzufassen. Die regionalen Unterschiede besitzen ziemlich sicher doch ein größeres Gewicht als es rein numerisch gesehen den Anschein macht.

\section{Bestimmung der Höhenlage der Jahresisotherme}

Leider ist es sehr schwierig, über die vertikale Temperaturverteilung im Alpenraum Aufschluß zu erhalten. Die nächsten aerologischen Stationen sind Mailand, München und Payerne. Von ihnen aus kann kaum in das Alpengebiet extrapoliert werden.

\section{Region}

Mittelbünden Berner Oberland Wallis verwendete Stationen

$$
\begin{aligned}
& \text { Weißfluhjoch - Jungfraujoch } \\
& \text { Grimsel - Jungfraujoch } \\
& \text { Grächen - Testa Grigia }
\end{aligned}
$$

Der Wert für Mittelbünden scheint etwas hoch, gibt doch Gensler (1967) für das Engadin 0.57\%/100 m an (1901-1940). Es war aber im Rahmen dieser Arbeit nicht möglich, das ganze Problem der Gradienten sorgfältiger zu prüfen, sondern die angegebenen Werte mußten als richtig angenommen werden.

Die damit gefundenen Werte für die Höhenlagen
Es mußte daher versucht werden, die Angaben aus den relativ wenigen Bergstationen zu gewinnen: In jedem Gebiet wurden von der jeweils höchsten Station aus Gradienten in Richtung der Jahresisotherme der klimatischen Schneegrenze bestimmt:

$\begin{array}{ll}\begin{array}{l}\text { Gradient } \\ { }^{\circ} \mathrm{C} / 100 \mathrm{~m}\end{array} & \text { Periode } \\ 0.62 & 1957-1966 \\ 0.58 & 1958-1966 \\ 0.55 & 1959-1966\end{array}$

der Jahresisothermen und damit der klimatischen Schneegrenzen in den drei Regionen sind in der folgenden Tabelle zusammengestellt. Die obere Angabe beruht auf der Annahme einer einzigen Regressionsgeraden für alle drei Gebiete, die untere basiert auf den regionalen Regressionskoeffizienten.

Die Höhenwerte sind auf \pm 50 m gerundet. 


\begin{tabular}{|c|c|c|c|c|c|c|c|c|}
\hline Region & $\begin{array}{l}\text { oberste } \\
\text { Station }\end{array}$ & Höhe & $\overline{\mathrm{T}}$ & Gradient & $\bar{\Gamma}$ an d. SG & $\triangle \mathrm{Hz} \cdot \mathrm{SG}$ & Höhe d. SG & Periode \\
\hline \multirow[t]{2}{*}{ Mittelbünden* } & W.-Joch & 2540 & -1.6 & 0.62 & -5.5 & 660 & 3200 & $1959-1966$ \\
\hline & & & -1.5 & 0.62 & -5.5 & 645 & 3200 & $1957-1966$ \\
\hline \multirow[t]{2}{*}{ Berrier Oberland } & Grimsel & 1956 & +1.4 & 0.58 & -5.5 & 1190 & 3150 & $1959-1966$ \\
\hline & & & +1.4 & 0.58 & -5.7 & 1225 & 3200 & $1958-1966$ \\
\hline \multirow[t]{2}{*}{ Wallis } & Zermatt & 1636 & +3.9 & 0.55 & -5.5 & 1710 & 3350 & $1959-1966$ \\
\hline & & & +3.9 & 0.55 & -6.0 & 1800 & 3450 & $1959-1966$ \\
\hline
\end{tabular}

* Alle Temperaturen der Station Weißfluhjoch (2667 m ü. M.) sind auf die Höhe des Versuchsfeldes (2540 $\mathrm{m}$ ü. M.), wo die Zahl der Tage mit

\section{Fehlergrenzen}

Mit welchen Fehlern sind die für die Höhe der klimatischen Schneegrenze angegebenen Werte behaftet? Um das richtig beurteilen zu können, müßte man vorerst genaue Angaben über die Fehler des Ausgangsmaterials haben, die aber leider nicht vorhanden sind. Es wurde ja schon darauf hingewiesen, da $\beta$ bei den Temperaturen ein systematischer Fehler wahrscheinlich ist, weil Werte, welche $2 \mathrm{~m}$ über Boden registriert wurden, Verwendung fanden. Ein gewisser Fehler muß auch bei der Festlegung der Zahl der Tage mit Schneedecke angenommen werden, denn es läßt sich nicht immer eindeutig entscheiden, ob mehr oder weniger als die Hälfte des Versuchsfeldes mit Schnee bedeckt ist. Vielleicht gleichen sich aber hier die positiven und negativen Abweichungen aus.

Um aber wenigstens eine Idee von der Größenordnung der möglichen Unsicherheiten zu erhalten, wurden Vertrauensgrenzen berechnet. Sie geben die Streuung des Regressionswertes y zu einem bestimmten $x$ an (vgl. Linder, 1960, p. 160 resp. 196). Beurteilt man die bei den graphischen Darstellun-
Schneedecke bestimmt wird, reduziert (Gradient $0.6 \% 100 \mathrm{~m}$ ).

gen angeschriebenen Vertrauensgrenzen, so fäll sofort auf, daß sie bei den einzelnen Gebieten vie enger um die Regressionsgerade liegen als im Fall wo die drei Gebiete als Ganzes angesehen werden Benützt man daher die Regressionsgleichungen, um zu einer bestimmten Temperatur die zugehörige Zahl der Tage mit Schneedecke zu bestimmen, sc weist das Resultat eine viel größere Unsicherhei1 auf, wenn man nicht von den einzelnen Regionen: sondern von ihrer Zusammenfassung ausgeht. Es deutet dies wiederum darauf hin, daß es eben doch richtiger ist, die drei Gebiete einzeln zu betrachten. Aus den Regressionsgleichungen ist ersichtlich. daß bei einer Temperaturabnahme von $1^{\circ} \mathrm{C}$ die $\mathrm{Zahl}$ der Tage mit Schneedecke um rund 20 zunimmt. was sehr gut mit Hess (1967) übereinstimmt (Koeffizient $\mathrm{c}$ in den Gleichungen 2 und $3.1^{\circ} \mathrm{C}$ Temperaturdifferenz wiederum bedingt eine Höhenänderung von rund $170 \mathrm{~m}$ (Gradient $0.6 \% / 100 \mathrm{~m}$ ). Kennt man nun die Unsicherheit der Zahl der Schneedeckentage, so läßt sich diese ohne weiteres in Metern ausdrücken. Für die Höhenlage der klimatischen Schneegrenze in den einzelnen Gebieten erhält man somit $(P=0.01)$ :

$$
\begin{array}{lll}
\text { Mittelbünden: } & 3200 \mathrm{~m} \pm 10 \mathrm{~m}( \pm 1 \text { Tag }) \text { Gradient } 0.62 & (1957-1966) \\
\text { Berner Oberland: } & 3180 \mathrm{~m} \pm 20 \mathrm{~m}( \pm 2 \text { Tage }) \text { Gradient } 0.58 & (1958-1966) \\
\text { Wallis: } & 3440 \mathrm{~m} \pm 30 \mathrm{~m} \text { ( } \pm 3 \text { Tage }) \text { Gradient } 0.55 & (1959-1966)
\end{array}
$$

Nimmt man die drei Regionen zusammen, so beträgt der Schwankungsbereich $\pm 140 \mathrm{~m}( \pm 18$ Tage, Gradient 0.6\%/100 m, 1959-1966).

10. Die Beziehung Höhe - Zahl der Tage mit Schneedecke

$\begin{array}{llll}\text { Region } & \text { Koeffizienten } & \text { Höhe der SG } & \text { Periode } \\ \text { Mittelbünden } & \mathrm{d}=19.23 & 3390 \mathrm{~m} & 1957-1966 \\ \text { Berner Oberland } & \mathrm{e}=0.10 & & \\ \text { Wallis } & \mathrm{d}=13.83 & 3220 \mathrm{~m} & 1958-1966 \\ \text { alle 3 Gebiete gemeinsam } & \mathrm{e}=0.11 & & \\ & \mathrm{~d}=-12.69 & 3670 \mathrm{~m} & 1959-1966 \\ & \mathrm{e}=0.10 & & \\ & \mathrm{~d}=-3.55 & 3260 \mathrm{~m} & 1959-1966 \\ & \mathrm{e}=0.11 & & \end{array}$


Weil Temperatur und Höhe stark miteinander korreliert sind (s. oben), scheint es nur vernünftig, eine Regression: Höhe (nicht Temperatur) - Zahl der Tage mit Schneedecke zu verwenden. Das entsprechende Modell ergab folgendes Ergebnis:

Zahl der Tage mit Schneedecke $=\mathrm{d}+\mathrm{e} . \mathrm{H}(\mathrm{H}$ in $1000 \mathrm{~m}$ )

Abgesehen vom Berner Oberland weichen diese Resultate stark von den oben angegebenen Werten ab. Obschon Zingg (1954) und auch Hess (1967) durchaus befriedigende Ergebnisse erhalten, muß man doch feststellen, daß die Höhe viel weniger Information enthält als die Temperatur. Uber die vertikale Schichtung der Atmosphäre (besonders bei Inversionen) gibt nur die Temperatur exakte Auskunft. Trotz der hohen Korrelation Höhe - Temperatur $(r=-0.95)$ vermag die Höhe die letztere nicht einfach zu ersetzen. Deshalb wird ein Rest der Streuung der Temperatur (ca. 10\%) von der Regression nicht erfaßt. Weil also die Höhe der Temperatur nicht völlig aequivalent ist, kann eine Regression Höhe - Zahl der Schneedeckentage für das vorliegende Problem nicht gleichgute Resultate liefern wie eine Regression Temperatur — Zahl der Tage mit Schneedecke.

Kurz sei hier auch die Frage der Gemeinsamkeitskorrelationen (Koller, 1963) angetönt: Die Höhenzunahme bewirkt ursächlich, daß die Temperatur abnimmt. Die Zahl der Schneedeckentage hängt ursächlich von der Temperatur ab. Somit beeinflußt die Höhe sowohl die Temperatur als auch die Anzahl der Tage mit Schneedecke. Es liegt also bis zu einem gewissen Grade eine Gemeinsamkeitskorrelation vor. Dennoch darf aber von einem echten kausalen Zusammenhang Temperatur - Zahl der Tage mit Schneedecke gesprochen werden. Deshalb ist auch die entsprechende Regression (unter Weglassung der Höhe) zulässig.

\section{Vergleich mit Literaturwerten}

Weil bisherige Schneegrenzbestimmungen in der Regel von Beobachtungen auf Gletschern ausgingen, lassen sich diese Angaben nur schwer mit den hier gegebenen vergleichen, die auf unvergletscherten Versuchsfeldern basieren. Wie die folgende $\mathrm{Zu}-$ sammenstellung zeigt, beträgt der Unterschied zu Höhenangaben, die man in der Literatur findet, i. a. $300-500 \mathrm{~m}$.

$\begin{array}{lll}\text { Kurowski (1891) } & \text { Finsteraarhorngruppe } & 2950 \mathrm{~m} \\ \text { Zeller (1892) } & \text { Triftgebiet } & 2750 \mathrm{~m} \\ \text { Jegerlehner (1902) Säntis } & 2450 \mathrm{~m} \\ & \text { Silvretta } & 2900 \mathrm{~m}\end{array}$

\begin{tabular}{clr} 
& Bernina & $2960 \mathrm{~m}$ \\
& Triftgruppe & $2750 \mathrm{~m}$ \\
& Finsteraarhorn & $2950 \mathrm{~m}$ \\
& Monte Rosa & $3260 \mathrm{~m}$ \\
Streiff-Becker & Säntis & $2450 \mathrm{~m}$ \\
(1947) & Clariden & $2650 \mathrm{~m}$ \\
& Monte Rosa & $3200 \mathrm{~m}$ \\
Hermes (1955) & N- und E-Rand \\
& \multicolumn{2}{c}{ der Alpen ca. 2500 m } \\
& Monte Rosa 3200-3300 m \\
Ambühl (1961) & Gotthard & $2800 \mathrm{~m}$ \\
Messerli (1967) & Säntis & $2700 \mathrm{~m}$ \\
& Jungfraujoch & $2700 \mathrm{~m}$ \\
& Wallis & $3100 \mathrm{~m}$ \\
Zingg (1954) & Prättigau & $3200 \mathrm{~m}$
\end{tabular}

\section{Zusammenfassung}

Die vorliegende Arbeit basiert auf der SchneegrenzDefinition von Zingg (1954): «Die Schneegrenze ist die Linie, jenseits der im Verlauf längerer Zeit mehr Schnee fällt als abschmilzt oder verdunstet ... Betrachtet man den Zeitraum einiger Jahre oder gar Jahrzehnte, so handelt es sich um die klimatische Schneegrenze unter der Bedingung, daß sich die Beobachtungen auf ein horizontales Feld normaler Exposition beziehen.» Es darf also nicht vergletschert sein.

Die Untersuchungen wurden zuerst mit den Stationen Vättis, Davos, Arosa, Weißfluhjoch (zusammengefaßt unter dem Begriff "Mittelbünden») ausgeführt und dann auf das Berner Oberland (Grindelwald, Guttannen, Grimsel) und das Wallis (Visp, Montana, Zermatt) ausgedehnt.

Die Grundlagen der Berechnungen bildeten Jahresmittelwerte der Temperatur, Jahressummen der Niederschläge (in mm Wasser) und Jahressummen der Tage mit Schneedecke.

Mit Hilfe der Regressions- und Korrelationsrechnung konnte in allen drei Gebieten nachgewiesen werden, daß die Zahl der Schneedeckentage überwiegend von der Jahresmitteltemperatur abhängig ist. Der Niederschlag (Jahressumme) übt nur einen verschwindend kleinen Einfluß aus. Durch Extrapolation der Regressionsgerade Temperatur (Jahresmittel, unabhängige Variable) - Zahl der Tage mit Schneedecke (Jahressumme, abhängige Größe) erhält man diejenige Jahresmitteltemperatur, welche notwendig ist, damit der Schnee 365 Tage liegen bleibt. Sie entspricht der Temperatur an der Schneegrenze.

Daraus läßt sich die Höhenlage der klimatischen Schneegrenze berechnen. Für die drei erwähnten Gebiete wurden folgende Werte gefunden (auf \pm $50 \mathrm{~m}$ gerundet): 


\section{Region}

Mittelbünden

Berner Oberland

Wallis
Jahrestemperatur an der $\mathrm{SG}$ in ${ }^{\circ} \mathrm{C}$

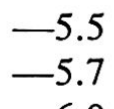

$-6.0$
Höhe der

$\mathrm{SG}$ in $\mathrm{m}$
Periode

3200

3200

3450
$1957-1966$

1958-1966

1959-1966
Vergleicht man diese Zahlen mit Höhenangaben aus der Literatur, so zeigen sich (abgesehen von Zingg, 1954) erhebliche Abweichungen. Sie beruhen vor allem darauf, daß in der Regel den Untersuchungen Beobachtungen auf Gletschern zugrunde lagen. Weil also diese Höhen, die man in der Literatur findet, i. a. auf einer anderen Definition der klimatischen Schneegrenze basieren, ist es ziemlich schwierig, sie mit den hier angegebenen Resultaten zu korrelieren.

Man müßte nun versuchen, ähnliche Berechnungen in außeralpinen Regionen durchzuführen, um zu sehen, ob die gefundenen Zusammenhänge auch dort Gültigkeit haben.

\section{Literaturverzeichnis}

Ahlmann, H. W. (1924): Le niveau de glaciation comme fonction de l'accumulation d'humidité sous forme solide. Geogr. Annaler, 3-4/1924, p. 223272.

Ambühl, E. (1961): 100 Jahre Einschneien und Ausapern in Andermatt (1860-1960). Die Alpen, 1961, p. 213-236.

Escher, H. (1969): Die Bestimmung der klimatischen Schneegrenze. Diplomarbeit, Geogr. Inst. Univ. Zürich (unveröffentlichtes Manuskript).

Gensler, G. (1967): Temperatur, Gletscherveränderungen und Firnbildung im Engadin. Wasser- und Energiewirtschaft, 6-7/1967, p. 207-212.

Hermes, K. (1955): Die Lage der oberen Waldgrenze in den Gebirgen der Erde und ihr Abstand zur Schneegrenze. Diss. Köln. Kölner Geogr. Arbeiten, Heft 5, 1955.

Hess, M. (1967): Versuch der Unterscheidung und Charakteristik der klimatischen Höhenstufen am Beispiel der polnischen Westkarpaten. Pet. Geogr. Mitt. 1/1967, p. 1-12.

Heinkes, H. und Untersteiner, N. (1952/1953): Wärmeumsatz und Ablation auf Alpengletschern. I: Geogr. Annaler, 1-2/1952, p. 99-158. II: Geogr. Annaler, 2/1953, p. 116-140.

Hoinkes, H. (1955): Über den Wärmehaushalt horizontaler Gletscherflächen in den Alpen. Met. Abh. des Inst. f. Met. und Geophysik der F. U. Berlin, Band II, Heft 4, p. 53 ff.

- (1968): Wir leben in einer Eiszeit. Umschau in Wissenschaft und Technik, Heft 26, p. 810-815.
Jegerlehner, J. (1902): Die Schneegrenze in den Gletschergebieten der Schweiz. Gerland's Beiträge zur Geophysik, Bd. V., Heft 3, Leipzig, 1902.

v. Klebelsberg, R. (1948/49): Handbuch der Gletscherkunde und Glaziologie. 2 Bände, Springer Wien.

Koller, S. (1963): Typisierung korrelativer Zusammenhänge. Metrika, Band 6, 1963.

Kreyszig, E. (1968): Statistische Methoden und ihre Anwendungen. Vandenhoek und Ruprecht, Göttingen.

Kuhn, W. (1958): Der Firnzuwachs pro 1957/1958 in einigen schweizerischen Firngebieten. Vierteljahresschrift der Naturf. Ges. Zürich. Schlußheft 1958, Jahrgang 103.

Kurowski, L. (1891): Die Höhe der Schneegrenze mit besonderer Berücksichtigung der Finsteraarhorngruppe. Penck Geogr. Abh., Band 5, 1891, p. $119-160$.

Linder, A. (1960): Statistische Methoden für Naturwissenschafter, Mediziner und Ingenieure. 3. Auflage, Birkhäuser Basel und Stuttgart.

Messerli, B. (1966): Die Schneegrenzhöhen in den ariden Zonen und das Problem Glazial-Pluvialzeit. Mitt. Natf. Ges. Bern, neue Folge, Band 23, p. 117 -148 .

- (1967): Die eiszeitliche und gegenwärtige Vergletscherung im Mittelmeerraum. Geogr. Helvetica, $3 / 1967$, p. $105-228$.

Schweiz. Meteorologische Zentralanstalt (MZA): Annalen der MZA, seit 1864.

- Unveröffentlichte Beobachtungen und Zusammenstellungen.

Seiffert, H. (1950): Das Klima an der Schneegrenze. Diss. Göttingen.

Streiff-Becker, R. (1947): Die Schneegrenze in den Alpen. Leben und Umwelt, 7/1947, p. 107-110.

Weber, E. (1967): Grundlagen der biologischen Statistik. 6. Auflage, Fischer Stuttgart.

Zeller, H. R. (1892): Die Schneegrenze im Triftgebiet. Geogr. Inst. Univ. Bern.

Zingg, Th. (1954): Die Bestimmung der klimatischen Schneegrenze auf klimatologischer Grundlage. Angewandte Pflanzensoziologie (Festschrift für Erwin Aichinger), II/1954.

- (1965): Schneeverhältnisse in den Schweizer Alpen, Winterbericht des Inst. für Schnee- und Lawinenforschung, Weißfluhjoch-Davos, Nr. 30, 1965/1966. 
1 Versuchsweise wurden in den nachfolgenden Berechnungen dennoch die Jahresmitteltemperaturen durch die Jahressummen der positiven Temperaturen ersetzt. Die Extrapolation der erhaltenen Regressionskurven zeigte aber, daß erst bei Temperatursummen, die im Bereiche von negativen Werten liegen, 365 Schneedeckentage erreicht werden, während theoretisch eine positive Jahressumme zu erwarten wäre. Es ist bis jetzt nicht klar, wieso das Resultat so unerwartet ausfällt.

2 Verschiedene theoretische Überlegungen lassen vermuten, daß ab ca. $2800 \mathrm{~m}$ ü. M. nicht mehr mit einer Geraden, sondern mit einer Kurve extrapoliert werden sollte. Man hat aber über ihren genauen Verlauf keinerlei Anhaltspunkte, da bis heute Meßdaten aus 2800-3000 m ü. M. fehlen. Es blieb daher nichts anderes übrig, als dennoch die berechneten Geraden zu verwenden.

\section{Rezensionen - Comptes rendus critiques}

\begin{abstract}
Bischoff, Gerhard: Die Welt unter uns. Welt- und Länderkunde aus der Vogelperspektive. 388 Seiten, 86 teils farbige Tafeln, 60 Skizzen. Safari-Verlag. Leinen.
\end{abstract}

Das vorliegende Buch erhebt keinen Anspruch, ein vollständiges Erdkundebuch zu sein, sondern zeigt an ausgewählten Beispielen, daß der Flugreisende von heute sich mit dem Blick aus dem Kabinenfenster der großen Überseeflugzeuge ebenso wie der kleineren Inlandmaschinen erhebliche Kenntnisse von den Ländern der Erde und dem Werk der Menschen verschaffen kann. So umschreibt der Verfasser seine Länderkunde aus der Vogelperspektive. Wir lernen die Erde von oben herab aufmerksam und verständnisvoll zu betrachten und erfahren, wie es mit der Kamera möglich ist, mit geradezu unglaublicher Präzision die feinsten Details auf der Erde einzufangen. Darüber hinaus werden durch die Luftaufnahmen dem menschlichen Auge Informationen vermittelt, die ihm sonst nicht zugänglich wären. Wir erfahren auch vieles über andere Wissensgebiete, z. B. Archäologie, Klimakunde, Landwirtschaft, Geschichte, Landesplanung und darüber, wie sie die Luftaufnahme in ihrem Fach verwenden und auswerten.

Das Buch sei somit nicht nur all jenen empfohlen, die in nächster Zeit eine Flugreise antreten können, sondern auch jenen, die Interesse an einer Auswahl vorzüglicher und gut interpretierter Flugaufnahmen aller Kontinente haben.

G. Schumacher

Lauer, Wilhelm (Herausgeber): Marburg und Umgebung. Ein landeskundlicher Exkursionsführer. 204 Seiten, 44 teils farbige Abbildungen und Karten. Geographisches Institut der Universität. Marburg 1967.

Am Geographischen Institut der Universität Marburg herrscht der schöne Brauch, die neuimmatri- kulierten Studenten durch Exkursionen in die Landschaft ihrer Studienstadt einzuführen, ein Brauch, dem auch an andern Hochschulen und für alle Studenten Nachachtung gebührte. Der bereits nach einem knappen Jahr in 2. Auflage erschienene Exkursionsführer, ursprünglich die Festgabe zum Geburtstag C. Schotts, bietet als Gemeinschaftswerk der Marburger Geographen, Geologen und Historiker hiefür die denkbar beste, musterhafte Grundlage, zur Einführung selbst wie zur Vertiefung, zumal es sowohl für den Fachmann als auch für den Laien geschrieben ist. Den Hauptteil machen Ausführungen über Altstadt und Stadt der Gegenwart (I. Leister), über Klima, Hydrographie, Natur- und Kulturlandschaft der Umgebung (W. Eriksen, M. Born, K. Scharlau) aus, eine beinahe erschöpfende Stadtgeographie einschließlich Umweltlehre bildend. Seitenmäßig zurücktretend präsentieren sich die 5 Exkursionsbeschreibungen, die wohl etwas wenig auf die übrigen Texte zurückgreifen, dagegen durch Routenkarten gut erfaßbar sind, namentlich auch deshalb, weil sie dem Wanderer jene Stellen zeigen, an denen Überblicke Einsichten in das Landschaftsgefüge ermöglichen. Das Buch ist überhaupt reichlich mit Karten und Graphika versehen, wenn auch zu bedauern bleibt, daß ihm keine Photos beigegeben werden konnten und - kein Taschenformat des Buches gewählt wurde. Trotz diesen Bemerkungen ist das sonst handliche Werk zweifellos ein Muster für ähnliche Unternehmungen und darf deshalb auch ausländischen Kollegen zur Benützung lebhaft empfohlen werden. E. Kern

Marti-Wehren, Robert: Im Saanenland. 2. Aufl. Stalder, Ernst Wilhelm: Das Schwarzenburgerland. Saurer, Emil: Twann. Berner Heimatbücher. Je 32 Bildtafeln. Verlag Paul Haupt, Bern.

Der Berner Verlag Paul Haupt hat seinerzeit, vor über 25 Jahren, die Initiative zur Herausgabe der 